\title{
Characteristics of Ammonia Spray Injected by Pressure-Swirl Atomizers
}

\author{
Yong Fan*1, Mitsuaki Ohtomo², Shunsuke Kasuga ${ }^{3}$, Norihiko Iki $^{4}$, Osamu Kurata ${ }^{1}$, \\ Taku Tsujimura ${ }^{4}$, Hirohide Furutani ${ }^{4}$ \\ ${ }^{1}$ Research Institute for Energy Conservation, National Institute of Advanced Industrial \\ Science and Technology (AIST), Tsukuba, Japan \\ ${ }^{2}$ Mechanical Division 1, Toyota Central R\&D Labs., Nagakute, Japan \\ ${ }^{3}$ Frontier Research Center, Toyota Motor Corporation, Susono, Japan \\ ${ }^{4}$ Renewable Energy Research Center, National Institute of Advanced Industrial Science and \\ Technology (AIST), Koriyama, Japan \\ ${ }^{*}$ Corresponding author email : yong.fan@aist.go.jp
}

\begin{abstract}
Ammonia $\left(\mathrm{NH}_{3}\right)$ is a potential carbon-free energy carrier for the storage, transportation, and utilization of energy produced from renewable sources. Ammonia can be used as a liquid fuel for spray combustion in industrial furnaces, engines, and gas turbines. Spray characteristics of liquid ammonia injected by commercial pressure-swirl atomizers (Delavan type WDA nozzles) have been investigated and compared with ethanol through shadowgraph images. The injection is driven by vapor pressure in the ammonia cylinder. Since ammonia has a boiling point lower than the room temperature, decompression evaporation of ammonia liquid may occur due to pressure drops both in the fuel supply line and across the spray nozzle. To avoid evaporation before the spray nozzle, tubes upstream were cooled down in the experiment. As a result, ammonia sprays with the hollow cone were formed. Unlike the ethanol spray in which the atomization into droplets were clearly observed, ammonia droplets evaporate while the atomization proceeds. Unique evaporating spray patterns were observed for ammonia. It was also found that the spray cone angle of ammonia is larger than that of ethanol, and then the catalog value of water with the $30^{\circ}$ nozzle. On the other hand, the measured cone angles of them show no great difference with the $80^{\circ}$ nozzle. Flow rate roughly correlates with injection pressure for ammonia, ethanol and water.
\end{abstract}

\section{Keywords}

Ammonia, Evaporating spray, Pressure-swirl atomizer, Spray cone

\section{Introduction}

Replacing a significant percent of fossil fuel energy with renewable energy is important in the fight against global warming. However, most renewable energy such as wind, solar and geothermal is typically intermittent and regional. Storage and transportation of the energy in battery or chemical forms are necessary for the utilization in urban areas with a high energy consumption. Both hydrogen $\left(\mathrm{H}_{2}\right)$ and ammonia $\left(\mathrm{NH}_{3}\right)$ are considered as potential carbon-free energy carriers for renewable sources. Hydrogen has unsolved challenges in safety and economic storage and transportation. On the other hand, mature technology for industrial scale production, storage and transportation of ammonia has been developed in its long history serving as the raw material of agricultural fertilizer.

Ammonia can be used as a liquid fuel for spray combustion in industrial furnaces, engines, and gas turbines [1-5]. Okafor et al. investigated the flame stability and emission characteristics of cofiring liquid ammonia in a methane swirling flame for gas turbine application [5]. In gas turbine burners, pressure-swirl atomizers are widely used. Spray 
characteristics such as spray cone angle, penetration length, droplet size and distribution have a great influence on the combustion [6]. The objective of the present study is to investigate the spray characteristics of liquid ammonia injected by pressure-swirl atomizers.

\section{Physical Properties of Ammonia}

Physical properties of ammonia, ethanol, water and diesel oil are listed in Table 1. Boiling temperature and latent heat are properties related to evaporation. Unlike ethanol, water and diesel oil which are liquid at $1 \mathrm{~atm}$, ammonia has a boiling point as low as $-33.3^{\circ} \mathrm{C}$. It is usually stored in liquified gas cylinder and supplied under a typical vapor pressure of $0.85 \mathrm{MPa}$ at normal temperature $\left(20^{\circ} \mathrm{C}\right)$. Without pressure rising pump or cooling of the supply line, decompression evaporation of ammonia liquid may occur in the supply line due to pressure drops or temperature rise. When liquid ammonia is injected from the spray nozzle into a chamber where the pressure is less than the saturation pressure at the chamber temperature, the so-called flash boiling occurs. Flash boiling of other fuels has been studied previously with single-hole and multi-hole injectors [7-9]. With flash boiling, atomization and evaporation are improved in the aspect of smaller droplet size and wider radial spread. On the other hand, the penetration length is reduced.

Density, surface tension and viscosity are properties related to atomization. In the spray experiment of present study, ethanol is chosen as the reference fluid for ammonia as they have similar surface tension. However, the viscosity of ammonia is almost one order lower than that of ethanol, which makes it easier to be atomized.

Table 1. Fluid properties.

\begin{tabular}{|l|c|c|c|c|}
\hline & Ammonia & Ethanol & Water & Diesel \\
\hline Boiling point at $1 \mathrm{~atm}\left({ }^{\circ} \mathrm{C}\right)$ & -33.3 & 78.4 & 100 & $180-230$ \\
\hline Density at $25^{\circ} \mathrm{C}\left(\mathrm{g} / \mathrm{cm}^{3}\right)$ & 0.60 & 0.79 & 1.00 & 0.83 \\
\hline Surface tension at $25^{\circ} \mathrm{C}(\mathrm{mN} / \mathrm{m})$ & 20.5 & 21.9 & 72.0 & 28 \\
\hline Kinematic viscosity at $25^{\circ} \mathrm{C}\left(\mathrm{mm}^{2} / \mathrm{s}\right)$ & 0.22 & 1.38 & 0.89 & 3.3 \\
\hline Viscosity at $25^{\circ} \mathrm{C}(\mu \mathrm{Pa} \cdot \mathrm{s})$ & 131.7 & 1082 & 890 & 2729 \\
\hline Latent heat at $25^{\circ} \mathrm{C}(\mathrm{kJ} / \mathrm{kg})$ & 1166 & 921 & 2442 & 250 \\
\hline
\end{tabular}

\section{Experimental Setup}

A schematic drawing of the experimental setup for ammonia and ethanol spray is shown in Fig. 1. As mentioned above, liquid ammonia is supplied from the cylinder by its vapor pressure. The injection pressure and hence the flow rate are regulated by a needle valve. The pressure is monitored by a pressure transducer and the flow rate is measured by a Coriolis mass flow meter (Bronkhorst, Model M15). In order to prevent evaporation of ammonia in the supply line, tubes are cooled by dry ice in ammonia experiments. Ethanol is supplied from a liquid tank by pressurized nitrogen gas.

Figure 2 shows the pressure-swirl atomizer (Delavan type WDA) used as the spray nozzle in the experiment, which is commercially available and widely used in industry applications. A swirling flow is produced while the flow is passing through the tangential slots, and a hollow cone spray is formed by the centrifugal force and vacuum zone in the center. In order to prevent the reverse flow of droplets, a rectified co-flow of air is fed to the chamber which carries the spray downstream. The air flow velocity is controlled within 1/10 of the liquid velocity to avoid serous influence on the spray. The No.5 (orifice diameter: $0.64 \mathrm{~mm}$, water flow rate: $14.5 \mathrm{~L} / \mathrm{h}$ at $0.5 \mathrm{MPa}$ ) and No.18 (orifice diameter: $1.32 \mathrm{~mm}$, water flow rate: $51.8 \mathrm{~L} / \mathrm{h}$ 
at $0.5 \mathrm{MPa}$ ) atomizers are used to examine the flow rate range dependence, and atomizers with reference spray cone angles of $30^{\circ}$ and $80^{\circ}$ for water are used to examine the spray cone angle dependence.

Instantaneous images of the spray in the chamber are captured by a high-speed camera (Photron SA-1) at $6000 \mathrm{fps}$. Shadowgraph method is employed. A point light source is formed by passing the cold lamp light through an iris. It is then shaped into parallel light by a convex lens to serve as the backlight for spray passing through the chamber. The shadowgraph image of spray is collected by the combination of another convex lens and camera lens.

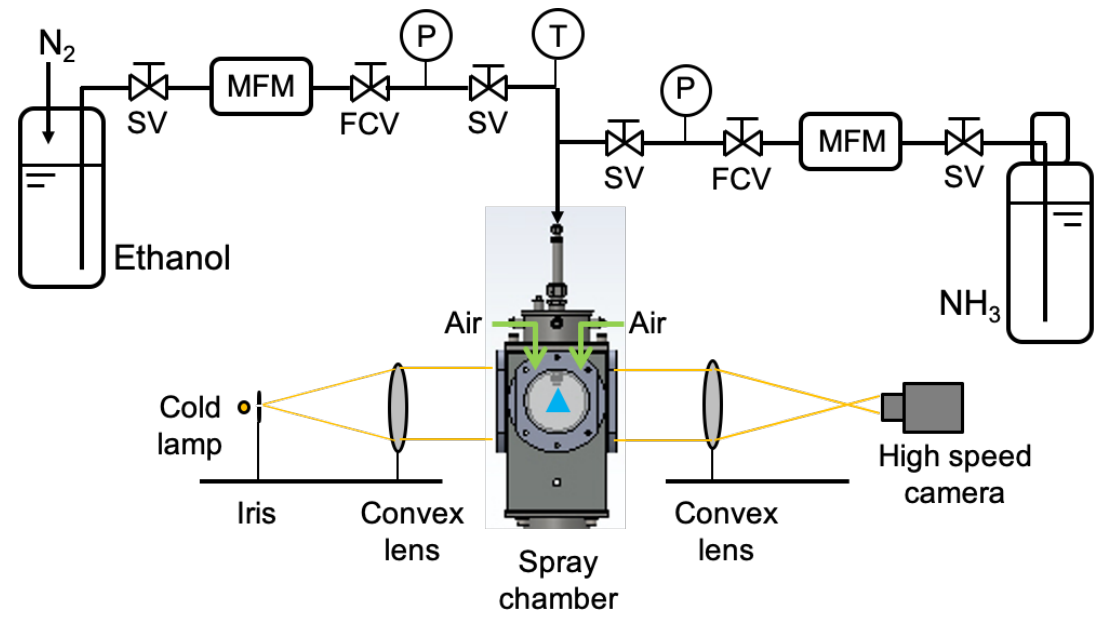

Figure 1. Experimental setup for ammonia and ethanol spray.

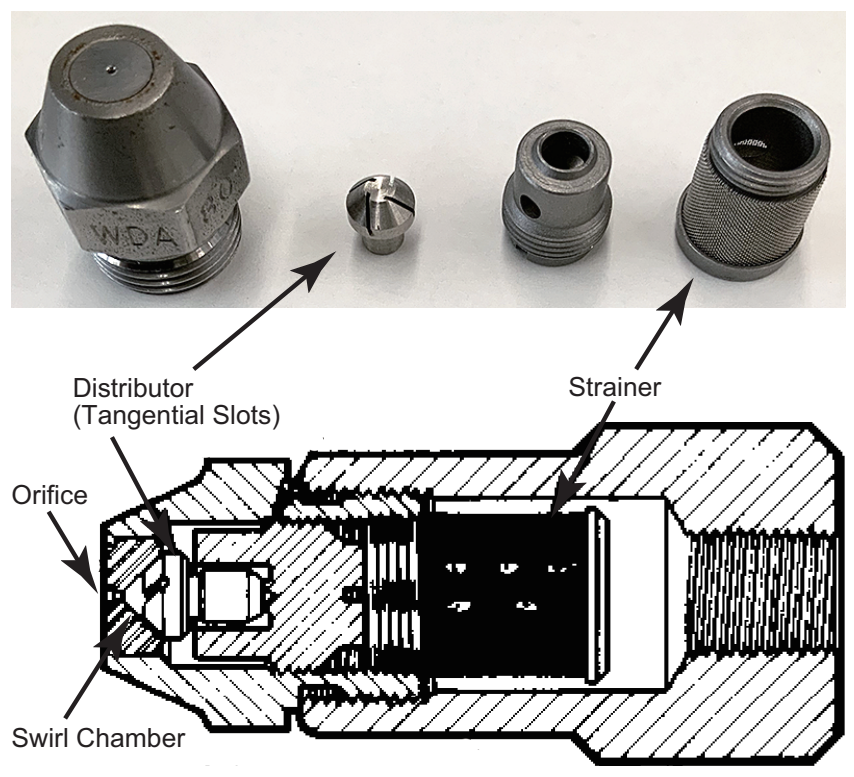

Type WDA (Hollow Cone) Pressure-swirl Atomizer

Figure 2. Pressure-swirl atomizer for spray experiment.

\section{Results and Discussion}

Figure 3 shows representative ethanol spray images with the No. 5 atomizers. Spray cones are formed. The spray cone angle with the $80^{\circ}$ atomizer is larger than that of the $30^{\circ}$ atomizer. Large droplets were observed. The pressure and flow rate are stable during the injection. 
(a) $30^{\circ}$

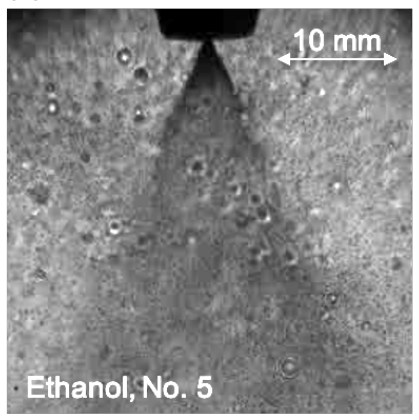

(b) $80^{\circ}$

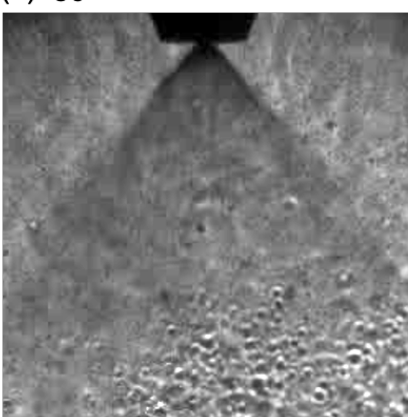

(c)

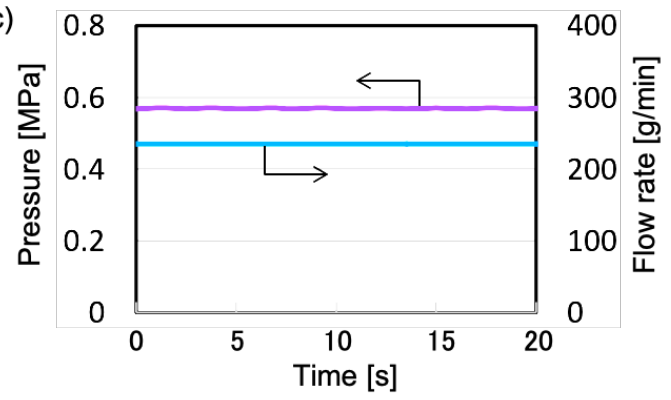

Figure 3. Ethanol spray images with Delavan WDA No. 5 atomizers.

(a) Case 1

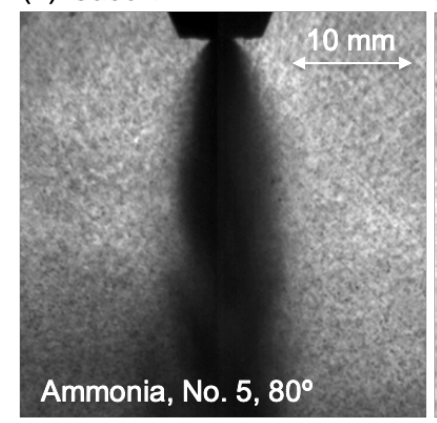

(b) Case 2

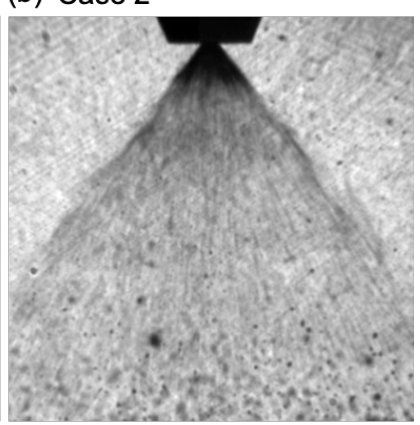

(c)

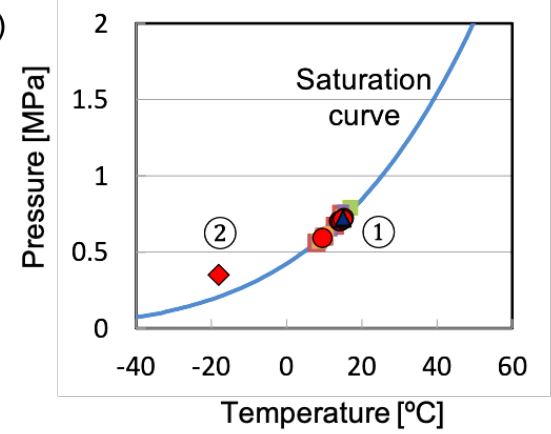

Figure 4. Ammonia spray images with/without cooling.

Figure 4 shows representative ammonia spray images with/without cooling of the ammonia supply line. When ammonia is supplied by vapor pressure in the cylinder without cooling, decompression evaporation of ammonia liquid may occur in both the supply line and the spray nozzle due to pressure drops. Measured pressure and temperature of ammonia for case 1 are located at the saturation curve, which shows the possibility of gas-liquid two-phase condition. Unlike the ethanol case, the flow rate of ammonia as well as the spray itself fluctuate (not shown here). The spray cone angle is obviously larger than $80^{\circ}$ and the spray is not well expanded downstream. As aforementioned, partial evaporation may occur before or inside the 
nozzle in this case. No visible droplet has been observed downstream, which shows the occurrence of evaporation together with the atomization.

On the other hand, when ammonia is injected at supercooled liquid condition (case 2) with the ammonia supply line being cooled by dry ice, the flow rate of ammonia as well as the spray itself become stable (not shown here). A normal spray cone is formed but it is unclear whether liquid film is formed as the conventional water spray is with the pressure-swirl atomizer. As compared to the ethanol spray in Fig. 3, droplets are overall smaller. This shows that ammonia is easier to be atomized and evaporated than ethanol, which is due to its smaller lower boiling point and viscosity.

Figures 5 and 6 shows ammonia spray images with the Delavan WDA No. 5 and No. 18 atomizers, respectively. Pressure and flow rate are stable. At the beginning of injection, a normal spray cone was observed. However, after a few seconds of injection, center part of the spray becomes darkish and blurry. It is considered that the evaporating spray observed here is caused by heat exchange with ambient gas in the chamber. As compared to No. 5 atomizer, droplets with the No. 18 atomizer are overall larger.

A comparison of measured spray cone angles for ammonia and ethanol and the catalogue value of spray cone angle for water is shown in Fig. 7. The Delavan WDA No. 5 atomizers with the spray cone angle of $30^{\circ}$ and $80^{\circ}$ for water are used. While the spray cone angles measured with the $80^{\circ}$ atomizer are almost the same for the three kinds of fluids, the spray cone angle with the $30^{\circ}$ atomizer has the largest value for ammonia, then ethanol, and the smallest one for water. As compared to ethanol, ammonia has the larger spray cone angle with the $30^{\circ}$ atomizer probably due to the smaller viscosity of ammonia. As compared to water, ethanol has the larger spray cone angle with the $30^{\circ}$ atomizer probably due to the smaller surface tension of water. However, those differences in surface tension and viscosity are not as important when the centrifugal force is strengthened with the $80^{\circ}$ atomizer.

(a) Right after injection

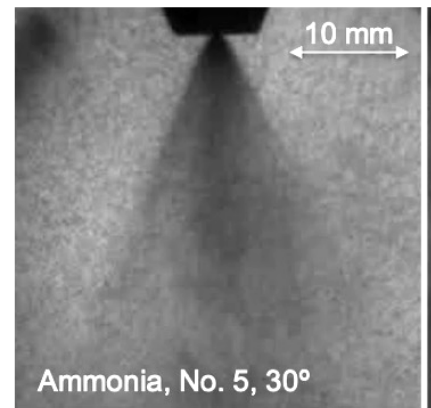

(c) (b) After a period of injection
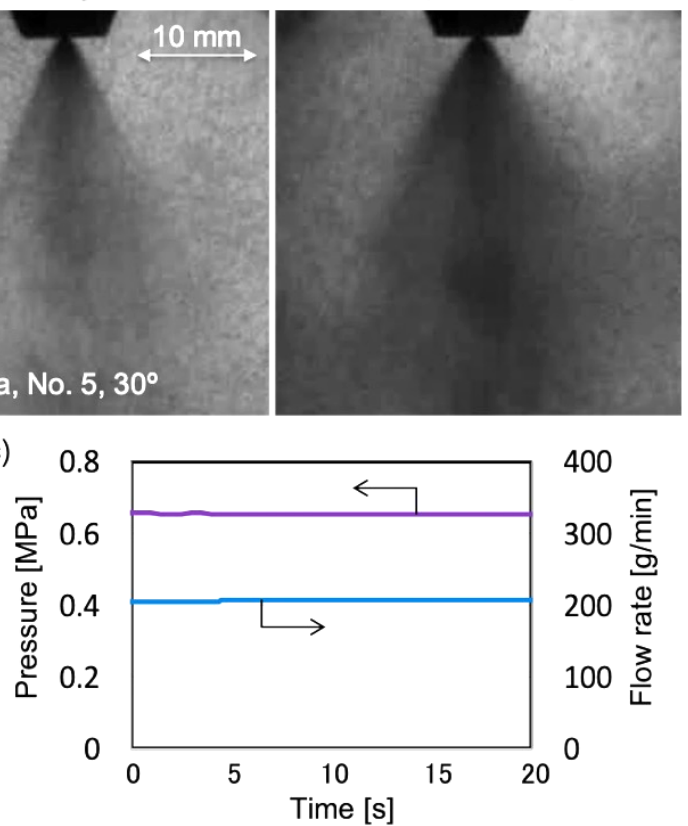

Figure 5. Ammonia spray images with Delavan WDA No. 5 atomizer. 
(a) Right after injection

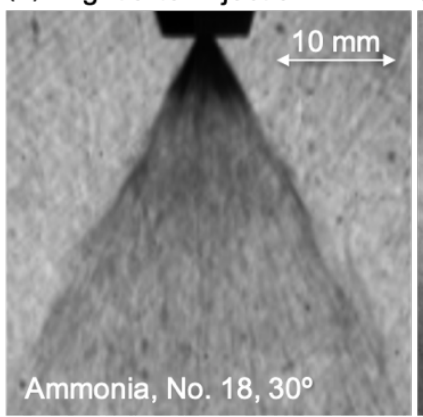

(c) (b) After a period of injection
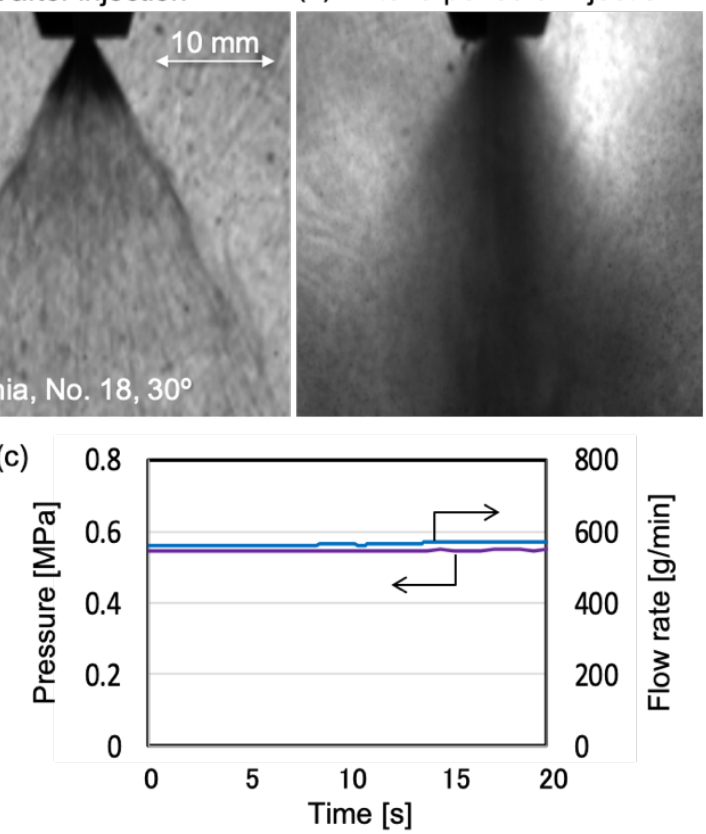

Figure 6. Ammonia spray images with Delavan WDA No. 18 atomizer.

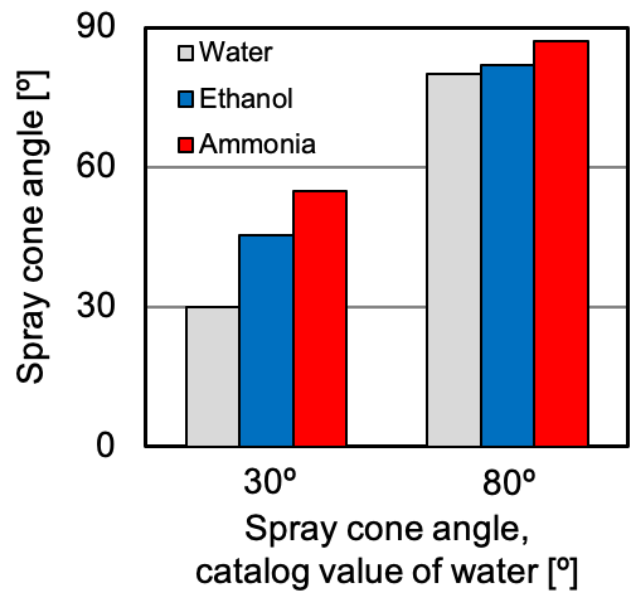

Figure7. Comparison of measured spray cone angles for ammonia and ethanol with Delavan WDA No. 5 atomizers

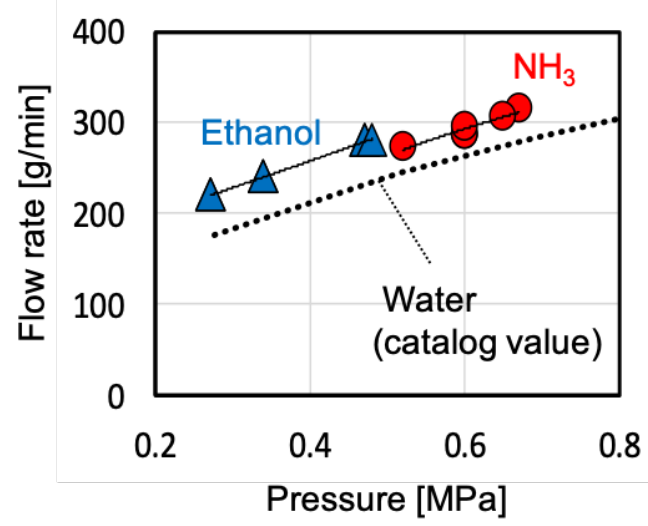

Figure 8. Comparison of ammonia and ethanol flow rates as functions of pressure. Delavan WDA No. 5 atomizer with a spray angle of $30^{\circ}$ is used. 
Figure 8 plots the ammonia, ethanol and water flow rates as functions of injection pressure. The Delavan WDA No. 5 atomizer with a spray angle of $30^{\circ}$ is used. It was found that both the flow rates of ammonia and ethanol increases with increasing the injection pressure as that of water does, though their flow rates are $10 \%$ larger.

\section{Conclusions}

Spray characteristics of ammonia and ethanol injected by pressure-swirl (Delavan type WDA) atomizers have been investigated through shadowgraph images. The conclusions are as follows:

- For ammonia, evaporating sprays were observed due to the low boiling point.

- With cooling of the tube upstream the nozzle, normal spray cone can be formed. However, droplets at the downstream evaporates after a period of injection.

- Spray cone angle of ammonia is larger than that of ethanol, and then the catalogue value of water with the $30^{\circ}$ nozzle. On the other hand, the measured cone angles of them show no great difference with the $80^{\circ}$ nozzle.

- Flow rate roughly correlates with injection pressure for ammonia, ethanol and water.

\section{References}

[1] Miyagawa, H., Suzuki, T., Ogasawara, K., Koike, M., Ammonia as a hydrogen storage and the feasibility for internal combustion engines, 19th world hydrogen energy conference, 2012. [2] Koike, M., Miyagawa, H., Suzuki, T., Ogasawara K., Ammonia as hydrogen energy carrier and its application to internal combustion engines, Sustainable vehicle technologies, 2012.

[3] Iki, N., Kurata, O., Inoue, T., Suzuki, M., Tsujimura, T., Furutani, H., Micro gas Turbine Firing Kerosene and Ammonia, ASME Turbo Expo 2015, GT2015-43689, 2015.

[4] Kurata, O., Iki, N., Inoue, T., Matsunuma, T., Tsujimura, T., Furutani, H., Kawano, M., Arai, K., Okafor, E.C., Hayakawa, A., Kobayashi, H., Development of a wide range-operable, richlean low-NOx combustor for NH 3 fuel gas-turbine power generation. Proceedings of the Combustion Institute, 37(4), 2019, 4587-4595.

[5] Okafor, E. C., Yamashita, H., Hayakawa, A., Somarathne, K. D. K. A., Kudo, T., Tsujimura, T., Uchida, M., Ito, S., Kobayashi, H., Flame stability and emissions characteristics of liquid ammonia spray co-fired with methane in a single stage swirl combustor. Fuel, 287, 2021, 119433.

[6] Fan, Y., Hashimoto, N., Nishida, H., \& Ozawa, Y., Spray characterization of an air-assist pressure-swirl atomizer injecting high-viscosity Jatropha oils. Fuel, 121, 2014, 271-283.

[7] Sher, E., Bar-Kohany, T., \& Rashkovan, A., Flash-boiling atomization. Progress in Energy and Combustion Science, 34(4), 2008, 417-439.

[8] Li, T., Dong, X., Hung, D. L. S., Li, X., \& Xu, M., Analysis of evaporation characteristics and heat transfer for flash-boiling sprays. International Journal of Heat and Mass Transfer, 127, 2018, 244-254.

[9] Xu, M., Zhang, Y., Zeng, W., Zhang, G., \& Zhang, M., Flash Boiling: Easy and Better Way to Generate Ideal Sprays than the High Injection Pressure. SAE International Journal of Fuels and Lubricants, 6(1), 2013, 137-148. 